\title{
Participación social en salud: un estudio de caso en Brasil*
}

\author{
Raquel Abrantes-Pêgo, M.en C.S.(')
}

\section{Abrantes-Pêgo R. Participación social en salud: un estudio de caso en Brasil. Salud Publica Mex I 999;4 I:466-474.}

\section{Resumen}

Objetivo. Estudiar la complejidad de la articulación entre poder público y población usuaria de los servicios de salud en Brasil, a partir de instancias locales de participación social, impulsadas por la descentralización de dichos servicios. Material y métodos. Se realizó un estudio cualitativo de los Grupos Ejecutivos Locales (GEL), en especial del localizado en una región periférica de la ciudad de Río de Janeiro, conocida como la AP.3.I. Durante 1986 se dio seguimiento a las reuniones del grupo y a sus actividades reivindicativas y de movilización. Se realizaron entrevistas abiertas con líderes de la comunidad, directivos de los servicios de salud y profesionales de instituciones universitarias que trabajaban en la localidad. El libro de actas del GEL fue considerado como una de las fuentes centrales de información. Resultados. El análisis permitió capturar la dinámica del juego entre la movilización y la respuesta de los servicios de salud al hacer resaltar, por un lado, los límites y potencialidades de las propuestas del gobierno en relación con la incorporación de los grupos sociales como interlocutores y actores propositivos en la definición de demandas y, por el otro, los marcos de la propia institucionalización de la participación popular en contextos caracterizados por la exclusión social y política. Conclusiones. Existe un desencuentro entre los formuladores de las políticas de salud y la población usuaria, pues se implementan estrategias de participación que no toman en cuentan las formas organizativas de los grupos sociales.

Palabras clave: participación comunitaria; salud; poder; Brasil

\section{Abrantes-Pêgo R. \\ Social participation in health: \\ a case study in Brazil.}

Salud Publica Mex 1999;4 I:466-474.

\section{Abstract}

Objective: To study the complex relationships between government power and health care utilization in Brazil, through the analysis of local initiatives of social participation fostered by decentralization of health services. Material and methods. A qualitative study was carried out among the Local Executive Groups (LEG), particularly the one located in the peripheral area of Rio de Janeiro, known as AP.3.I. Group activities were followed during 1986. Data were collected by means of open interviews to community leaders, health service managers and university faculty. One of the main information sources was the LEG proceedings book. Results. Data analysis disclosed the dynamics of the relationship between social mobilization and healthcare responses, underscoring the limits and potential of governmental initiatives regarding the inclusion of social groups as active interlocutors and actors in the definition of demands, as well as the limits of institutionalization of popular participation in settings socially and politically deprived. Conclusions. Contraposition between health policymakers and healthcare users is evidenced by the implementation of social participation strategies that do not take into account the organizational figures of social groups.

Keywords: community participation; health; power; Brazil

Este artículo se basa en una ponencia presentada en el "Encuentro Latinoamericano de Municipalización de la Atención Primaria a la Salud", realizado en Cuernavaca, Morelos, en septiembre de 1998, y en el trabajo de investigación para obtener el título de maestría en Ciencias Sociales "Identidades coletivas, democracia e políticas sociais -un estudo de caso na área da saúde", en el Instituto de Filosofia e Ciencias Sociais da Universidad Federal do Rio de Janeiro, 1992.

(I) Centro de Investigación en Sistemas de Salud, Instituto Nacional de Salud Pública, México.

Fecha de recibido: 15 de febrero de 1999 - Fecha de aprobado: 20 de septiembre de 1999 Solicitud de sobretiros: Raquel Abrantes Pêgo. Centro de Investigación en Sistemas de Salud, Instituto Nacional de Salud Pública. Universidad 655, colonia Santa María Ahuacatitlán, 62508 Cuernavaca, Morelos, México. Correo electrónico: mpego@insp3.insp.mx 
F 1 presente trabajo centra su atención en instanE cias locales de participación, creadas a partir del poder público, como un canal de articulación de intereses durante el proceso de descentralización del sistema de salud en Brasil. Esas instancias serán analizadas como un campo de acción política y social capaz de conformar nuevas demandas y dar visibilidad a nuevos actores sociales, y donde convergen autoridades, técnicos, partidos políticos y movimientos sociales, entre otros; por consiguiente, se trata de un campo dominado por conflictos de diversa naturaleza y por distintas relaciones de poder.

Investigamos los Grupos Ejecutivos Locales (GEL), en especial el localizado en una región periférica de la ciudad de Río de Janeiro, llamada Leopoldina y conocida, para efectos administrativos, como AP.3.1. La investigación abarca el periodo comprendido entre 1983 -cuando los grupos son creados- y 1989 -cuando se desmovilizan. Los GEL fueron instancias de participación popular previstas en el Programa de Acciones Integradas de Salud, que fue puesto en práctica al final del periodo militar (1983),,* así como en el Sistema Unificado y Descentralizado de Salud (SUDS) de 1985, durante el periodo de gobierno conocido como la Nueva República, que representa el final del periodo militar.

Las Acciones Integradas de Salud (AIS) marcaron el inicio del proceso de descentralización del sistema de salud en Brasil, y se realizaban mediante convenios trilaterales entre el Ministerio de Seguridad y Asistencia Social (que se encargaba de la asistencia médica de los derechohabientes), el Ministerio de la Salud

\footnotetext{
* En 1964, un golpe militar depone al presidente Goulart; una junta militar asume el poder por medio del jefe del Estado Mayor del Ejército, general Castelo Branco, quien completa el mandato de Goulart. El Congreso continúa funcionando con poderes limitados hasta 1968, cuando se clausura por medio de un "acto institucional", el AI-5, y lo reabren para elegir un nuevo presidente en 1969: el general Emílio Garrastazu Médici. Es el periodo más duro de represión política y coincide con altas tasas de crecimiento económico y de control salarial. En 1974 empieza el periodo de gobierno del general de reserva Ernesto Geisel, quien anuncia el inicio de un proceso de liberalización "lenta, gradual y segura" del régimen, que coincide con el fin del llamado "milagro económico" y la movilización de la sociedad civil en torno a cuestiones políticas y sociales. En 1985 inicia la Nueva República con la elección por el Congreso de un presidente civil, el liberal Tancredo Neves, quien cae gravemente enfermo y muere en víspera de la toma de posesión; lo sustituye el ex senador gobiernista y vicepresidente electo José Sarney, del partido Democrático Social. La Nueva República termina en 1989 con la primera elección directa para presidente, en la que Fernando Collor de Mello vence al candidato del Partido de los Trabajadores (PT), el obrero Lus Lula Inácio da Silva.
}

(responsable de actividades de prevención y de otorgar atención médica a la población pobre en general) y las Secretarías de Salud de los estados. Se buscaba promover la transferencia sistemática de recursos financieros a los estados y municipios, y estructurar instancias nacionales, estatales y municipales de gestión colegiada, para impulsar una participación social de carácter institucional en las políticas de salud. ${ }^{1,2}$ Dichas instancias estaban formadas por representantes del gobierno y de la sociedad (usuarios, trabajadores de salud y prestadores de servicios privados). La propuesta de crear comisiones era una respuesta a la necesidad de garantizar la integración de los gestores públicos entre sí y de éstos con el sector privado, esto es, a la necesidad de concertar intereses entre actores diversos.

La puesta en práctica de las AIS coincide con el inicio de nuevas administraciones municipales elegidas en 1982, comprometidas con una agenda social y con el proceso de democratización de la sociedad brasileña. Con la Nueva República, las AIS se transformaron en un eje fundamental de la política de salud y, a partir de 1985, con la creación del Sistema Único y Descentralizado de Salud (SUDS), se constituyeron en una importante estrategia para la universalización, la descentralización y la creación del Sistema Único de Salud (SUS), que prevé la participación de la comunidad, de acuerdo con el texto de la Constitución de 1988.

La AP.3.1 era una región administrativa compuesta por tres colonias y un número significativo de favelas (69), que en aquel momento representaban $18 \%$ del total de las existentes en la ciudad de Río de Janeiro. De ese total, 43 aglutinaban aproximadamente a 3000 habitantes cada una; otras 19 tenían de 3000 a 9000 individuos, y las siete restantes oscilaban entre 9000 y 15000 habitantes. Veintiocho por ciento de los habitantes de la AP.3.1 vivían en favelas y ocupaban $6.7 \%$ del área. Esa población trabajaba principalmente en el sector servicios (34.7\%) y en la industria de la transformación $(24.2 \%)$, con un ingreso promedio de dos salarios mínimos. Predominaba el grado de instrucción básico, y los problemas principales de salud estaban relacionados con las condiciones de vida de esta población.

\section{Material y métodos}

El GEL, como un campo de acción social y política, sirvió como objeto empírico de reflexión, referido a un ámbito más amplio como lo es la descentralización de los servicios de salud en Brasil. Para recuperar la experiencia social de participación en el GEL se consideraron: a) los conflictos a partir de los cuales se constituyó 
el GEL; b) las precondiciones estructurales a partir de las cuales la propuesta se gestó; c) los sujetos de esa experiencia, y d) su proceso de desarrollo. ${ }^{3-7}$ El método elegido de acercamiento al problema fue de naturaleza cualitativa y consistió en acompañar, de enero a diciembre de 1986, las reuniones del grupo y sus actividades reivindicativas y de movilización. ${ }^{3,8}$ Se realizaron entrevistas abiertas con los líderes de la comunidad, con algunos de los directivos de los servicios de salud y los profesionales de las instituciones universitarias que trabajaban en la localidad. El libro de actas del GEL fue considerado como una de las fuentes centrales de información, al igual que otros documentos de la organización; se consultaron también materiales propositivos sobre participación social elaborados por el Movimiento Popular por la Salud, que está compuesto por varias asociaciones vecinales, y, por último, documentación oficial de los ministerios involucrados y de la Secretaría de Salud del Estado, además de la bibliografía analítica sobre el tema.

Para la elaboración del análisis partimos del supuesto de que los alcances y límites de las formas comunitarias de organización están definidos por la capacidad del grupo de elaborar una identidad estructurada en el conflicto que le da vida y de organizarse para defender intereses o expresar voluntades; esta capacidad depende de los recursos de poder que el grupo controla, los que a su vez son definidos por el lugar que éste ocupa en la estructura social. Además, el análisis tomó en consideración el tipo de relaciones que se establecen con otros actores de la sociedad y del Estado, así como el tipo de articulación que se estructura con procesos político-sociales más amplios, en particular, con la transición hacia una sociedad más democrática en su expresión social. Por este motivo, para explicar la acción sociopolítica del GEL, en el presente estudio se procuró demostrar que la identidad de este grupo estuvo articulada a sus demandas sociales, a su capacidad de vocalizarlas ante el poder público y a la presencia de otros actores sociales que influyeron en el desarrollo de las políticas de salud.

\section{Resultados}

En el contexto de la transición, la salud, entendida como un problema social urgente, posibilitó la movilización de diferentes grupos sociales. Esas movilizaciones promovidas por médicos, estudiantes, salubristas, habitantes de la periferia y partidos de oposición, formaron parte del llamado proceso de "ebullición de la sociedad civil" ${ }^{\prime \prime 110}$ que tanto marcó a la transición brasileña. A partir de esas movilizaciones se cuestionaron: a) el crecimiento artificial del sector médico-hospitalario privado, inducido por el propio Estado al incluir nuevos segmentos sociales en la seguridad social sin aumentar la oferta de servicios públicos, estrategia que provocó el deterioro de tales servicios y la sobrevaloración de la atención privada; b) los pésimos salarios de los profesionales de la salud; c) la saturación de las instituciones públicas, expresada en largas filas y en una mala asistencia, y d) la falta de participación de los sectores sociales organizados en la determinación de las políticas sectoriales y en el control del uso de los recursos.

Ese movimiento febril de la sociedad civil combinaba agitaciones contra el gobierno militar y demandas sociales presentes en diferentes espacios de la sociedad. En el ámbito académico, los investigadores en salud pública se organizaron en torno del compromiso de producir un nuevo conocimiento que sirviera a las causas populares y fuera capaz de materializar una propuesta alternativa, basada en el principio de la salud como un bien público, esto es, como un derecho social y no como un bien privado, objeto de transacción en el mercado. De ese proceso surgió la propuesta de un sistema único de salud, público, universal, jerarquizado y regional, con vistas a resolver los problemas de salud de forma integral. ${ }^{11}$

Por otra parte, los departamentos universitarios de Medicina Preventiva, sectores de las iglesias, particularmente de la Católica, y los municipios gobernados por la oposición, representada inicialmente por el Movimiento Democrático Brasileño, promovieron experiencias alternativas de participación social. Estas experiencias estaban basadas en la participación popular en actividades de promoción de la salud. Muchas abarcaban desde la identificación del problema de salud y la toma de decisiones, hasta la formulación y la ejecución de acciones. ${ }^{12-13}$

A partir de esas experiencias y otras, emergió un movimiento llamado por su propios protagonistas de "reforma sanitaria" y se delineó una perspectiva de acción política con vistas a establecer nuevas relaciones entre el Estado y la sociedad, mismas que no se agotaban en transformaciones restrictivas impuestas al sistema político. Asimismo, se conformó un fuerte movimiento social entre los habitantes de las colonias pobres, organizados en asociaciones comunitarias o en clubes de madres, y que demandaban agua, electricidad, habitación y transporte; se sentían con el derecho de intervenir junto al gobierno para hacer valer sus derechos sociales y políticos.

En Río de Janeiro las asociaciones vecinales de diferentes colonias y favelas se organizaron en Federaciones -la Federación de Asociaciones de Moradores 
de Río de Janeiro (FAMERJ) y la Federación de Asociaciones de Moradores de Favelas de Río de Janeiro (FAFERJ). Estas organizaciones lograron promover iniciativas informales de participación, como los GEL, y surgieron como una alternativa a la falta de interés del poder público por modificar una situación caracterizada por carencia de servicios de agua, teléfono, iluminación, transporte y abastecimiento.

\section{La institucionalización del GEL correspondiente a la AP.3.I}

El estado de Río de Janeiro fue uno de los primeros en firmar un convenio con el gobierno federal en el marco de las AIS en 1983. En el convenio estaba prevista la creación de instancias colegiadas de participación que abarcaran diferentes ámbitos del proceso de decisión, como la Comisión Interinstitucional Municipal de Salud (CIMS), con el objetivo de coordinar el programa en el municipio de Río de Janeiro. Esta comisión quedó constituida por representantes de las instituciones involucradas en el convenio, esto es, por los titulares de la Secretaría de Salud del Estado de Río de Janeiro y de la ciudad de Río de Janeiro; además, en ella se incluyó a representantes del Ministerio de Seguridad y Asistencia Social, a través del Instituto Nacional de Asistencia Médica (INAMPS, por sus siglas en portugués) -órgano que responde por la asistencia médica-, y del Ministerio de Salud. También se creó el Consejo Estatal de Salud -como instancia de carácter consultivo al servicio de la Secretaría de Salud-, compuesto por diversos sectores, así como la Comisión Ejecutiva de Áreas de Planificación (CEAP); ésta última con la finalidad de dar operatividad a las propuestas y decisiones del GEL. La CEAP estaba integrada por todos los directivos de las unidades de salud del área y por representantes de la comunidad local, con criterios de representación paritaria.

\section{Quién participa}

Desde la perspectiva del gobierno del estado, esas instancias eran importantes, no tanto para garantizar una real participación de la comunidad, sino para legitimar el proceso de integración institucional y como espacio privilegiado de negociación en el ámbito local. Algunos funcionarios consideraban que para hacer viable políticamente la propuesta de descentralización, el GEL debía ser paritario, de tal forma que se constituyera en un instrumento capaz de garantizar un microespacio de demanda de la población y de facilitar la integración. Con una medida contraria se corría el riesgo de repetir la experiencia de Nova Iguaçu -ciudad de la periferia de Río de Janeiro-, donde había un Consejo Comunitario formado por 120 asociaciones, lo que podía crear restricciones por parte de los directivos de las unidades de salud.

Así, la principal preocupación de la Secretaría Ejecutiva de la CIMS, creada pocos meses después de firmado el convenio, fue la de constituir con rapidez los GEL y definir su composición, "a fin de evitar una participación tumultuosa de la comunidad", según una de las autoridades entrevistadas; cabe aclarar que los GEL ya funcionaban de manera informal y que las primeras reuniones habían resultado en verdaderas plenarias. La propuesta de algunas de las comunidades participantes era en el sentido de que la participación comunitaria tenía que darse desde las bases, para no comprometer el propio proceso de participación. A su vez, la FAMERJ consideraba que los representantes debían ser elegidos por ellas.*

Sin embargo, existía cierta tradición de lucha social en la AP.3.1; en el interior de la comunidad había un antecedente organizativo y de lucha ideológica debido a la presencia de partidos políticos y organizaciones de izquierda. A su vez, las luchas sociales por mejorar las condiciones ambientales ya se venían desarrollando en algunas colonias desde 1977, debido a la presencia de una fábrica de curtido de cuero responsable de un número significativo de enfermedades respiratorias, por la emisión constante de sustancias tóxicas. ${ }^{14}$

Algunas colonias y favelas tenían también sus propios consejos comunitarios, que eran espacios de discusión mensual en torno a las cuestiones de salud. Muchos participantes estaban a favor de una posición de autonomía frente al Estado. Esos consejos estaban integrados por los líderes de las asociaciones vecinales, de las favelas y de las comunidades, así como por militantes de las Comunidades Eclesiásticas de Base. Por otro lado, los profesionales de la salud, por medio de su práctica médica en las colonias pobres, intentaban promover la organización de las mismas, mientras que políticos, tanto del partido del gobierno como de la naciente oposición, operaban mediante la maquinaria política clientelista, particularmente en los periodos electorales.

Así, en las primeras reuniones para la creación del GEL de la AP.3.1 estaban presentes tanto representantes de asociaciones vecinales, de la FAMERJ y de la FAFERJ, como personas de la comunidad indepen-

* Reunión del día 28 de febrero de 1984, convocada por la CEAP. Libro de actas del GEL de la AP.3.1. 
dientes. Sin mucha dificultad se definió conjuntamente cuáles serían los principales puntos de actuación del GEL; sin embargo, no fue fácil determinar cómo participaría la población.

En las primeras reuniones, diversos residentes cuestionaron la propuesta del gobierno, en el sentido de que debía haber una participación paritaria de los distintos sectores, y lo sugerido fue abrir las reuniones del GEL a toda la comunidad. Para ellos, el GEL era "un espacio de discusión entre las instituciones y las comunidades" y debía ser utilizado como "instrumento de quejas" de la comunidad. Por eso, evidentemente, cuanta más gente participase, mejor. La participación paritaria, para ellos, sólo tenía sentido en el caso de que el GEL tuviese la facultad de deliberar por votación de la mayoría y no por consenso, dado que éste sería difícil de alcanzar. La FAFERJ apoyaba esta posición; en cambio, la FAMERJ consideraba que los representantes debían ser elegidos por los organismos de la Federación, de tal forma que se fortaleciera ésta y, en consecuencia, el GEL.*

Los residentes también expresaban una cierta desconfianza en relación con las verdaderas intenciones de la autoridades. Consideraban que la discusión era falsa, ya que la forma de participación comunitaria ya estaba predefinida, y lo que las autoridades procuraban era el respaldo de la población. Por medio de la FAMERJ, los residentes reclamaban que las asociaciones vecinales no habían sido convocadas desde el inicio de la formación del GEL, lo que había dificultado la promoción del mismo en la comunidad. ${ }^{\ddagger}$

Otro punto de conflicto fue la sustitución de los residentes por los representantes de las organizaciones existentes. Los residentes expresaban en todo momento un interés por participar directamente y sentían un cierto desengaño respecto a los representantes institucionalizados; ${ }^{\S}$ los criticaban en relación con sus actuaciones, definidas como de carácter esencialmente "reivindicativo", cuando, desde el punto de vista de los residentes, los representantes debían preocuparse por "organizar a las comunidades". A esta cuestión, los representantes aludidos respondieron que las "comunidades presentes tenían urgencias y que, por tanto, las demandas de puestos de salud y de saneamiento se mantenían".\&

\footnotetext{
* Relatoría del GEL -CEAP IV, 15 de diciembre de 1984.

‡ Reunión del 1 de marzo de 1984. Libro de actas del GEL de la A.P.3.1.

§ Reunión del Gel de 28 de febrero de 1984, del 28 de agosto de 1984 y 18 de marzo de 1989 .

\& Reunión del día 28 de agosto de 1984.
}

De hecho, el GEL nunca fue institucionalizado conforme proponía el gobierno. La comunidad no eligió a su representante, y la propia forma que toma el GEL hace que la designación de los delegados se torne secundaria. Los obstáculos que se interpusieron en el proceso de selección de los representantes se dieron, principalmente, debido a la gran variedad de formas de organización popular que convivían entre sí: consejos comunitarios, asociaciones vecinales, representantes de las federaciones, clubes de madres y otros; y en contrapartida eran 15 los representantes de los servicios de salud.

Asimismo, la falta de información y compromiso entre las autoridades del gobierno con este tipo de instancia de participación significó un obstáculo para la mayor participación de la comunidad. Los residentes se percataron de que era necesario conocer los presupuestos de las diversas instituciones del área y también garantizar un mayor compromiso de las direcciones de los servicios. Sin eso se tornaba imposible fiscalizar los convenios que los servicios públicos suscribían con el sector privado, evaluar los servicios, crear normas comunes para los mismos y desarrollar grupos de salud en la comunidad para promover debates sobre problemas de salud y para la educación en cuidados básicos.

Con el SUDS, ya en la Nueva República, se intensificaron todavía más las discusiones, en cuanto a quién participaría y cómo, entre una multitud de entidades y organizaciones populares, todas rivalizando entre sí por la medalla de la representación. Estaba prevista la creación del Consejo Municipal de Salud, instancia coordinadora del SUDS en Río de Janeiro, con poder de decisión sobre las acciones de salud. Esta estructura organizativa quedó integrada por una verdadera multitud: 23 representantes institucionales (de las secretarías de Desarrollo Social, Obras, Educación y Desarrollo Urbano, así como de universidades, del Ministerio de Salud y del INAMPS) y 23 de la sociedad civil (ocho representantes comunitarios del GEL, de la FAMERJ, y la FAFERJ), más otros 15 a definir por la Secretaría de Salud.

La entrada del GEL en el Consejo reanimó y complicó considerablemente la discusión en torno de la representación popular. Ahora el problema no era solamente saber quién votaba en el GEL, sino quién votaría para elegir a los delegados del GEL en una instancia exterior. En el primer nivel se debía definir si votarían todos los presentes, los presidentes de asociaciones o los líderes informales de la comunidad. La propuesta oficial, que restringía el voto a representantes y presidentes de asociaciones, fue la que ganó. Sin embargo, contribuyó para la desintegración del GEL como instancia de movilización y participación local. 


\section{La actuación del GEL}

No obstante, la paradoja descrita con anterioridad no puede ser interpretada como un obstáculo para la participación de la comunidad de Leopoldina. De hecho, desde sus primeras reuniones, la comunidad del GEL demandó centros médicos de atención y saneamiento; promovió movilizaciones contra la anulación del sector de urgencias del Hospital General de Bonsucesso; lanzó propuestas para ampliar la capacidad de atención del Hospital Nossa Senhora do Loreto, ${ }^{*}$ principalmente del servicio quirúrgico infantil, y se manifestó en contra de la puesta en marcha del Programa de Suplemento Alimenticio porque atendía a un número muy pequeño de habitantes.

La dinámica que tomaron las reuniones del GEL, donde convergían miembros de las comunidades y representantes del poder público, contribuyó a una doble instrumentación política. Fue una calle de doble sentido en la cual el interés del gobierno por encontrar apoyo en la población, fue correspondido por los intereses de la misma en el sentido de obtener ganancias, aunque limitadas. La comunidad sabía que los cargos de los directores de los servicios eran políticos. Por medio del GEL, los sectores populares procuraron limitar el uso político de los servicios públicos haciendo propuestas concretas, como aquella que promovía la participación del GEL en los cambios de dirección, de tal forma que sus puntos de vista fuesen por lo menos escuchados.

Se pueden distinguir dos modalidades de acción del GEL: una, de Grupos de Trabajo (GT), incentivados por la propia instancia gestora del Programa de AIS la CIMS-; la otra surge de las necesidades de los participantes y para dar al grupo más organicidad. Los primeros grupos de trabajo formados fueron el de salud de la mujer, del niño, otro de vigilancia epidemiológica y uno más relacionado con el presupuesto..$^{\ddagger}$

Para los GT fue importante la presencia de las dos instituciones universitarias que desarrollaban sus trabajos de enseñanza en esa área: la Escuela Nacional de Salud Pública (ENSP-FIOCRUZ) y la Facultad de Medicina de la Universidad Federal de Río de Janeiro. Los estudiantes de la ENSP, por ejemplo, realizaron junto con la comunidad un diagnóstico para subsidiar la actuación del GEL y el trabajo de vigilancia epidemiológica. Ambas instituciones tenían centros de atención en el área.

\footnotetext{
* Reunión del día 11 de mayo de 1984.

‡ Reunión del día 27 de julio de 1984.
}

El otro tipo de acciones que garantizaron la presencia de las personas de la comunidad en el GEL fueron las que surgieron de la propia percepción de carencia frente a los servicios de salud y de la posibilidad de formular demandas en torno a ellas. Fueron acciones de naturaleza colectiva, a partir de las cuales la población procuraba hacer valer sus derechos -como lo fue la lucha en torno de la reforma del sector de urgencias del Hospital General de Bonsucesso, de la inauguración del servicio de urgencias en el Instituto de Atención Infantil y de la reformulación de las actividades del Centro de Salud Américo Veloso. Cabe destacar, asimismo, que se elaboraban actas de todas las reuniones y que la comunidad luchó por garantizar su presencia en la mesa coordinadora del GEL. Esa lucha duró dos años hasta que se acordó que se invitaría a un representante de la comunidad, escogido por los presentes.*

Para ese tipo de acción, la comunidad presente en el GEL no esperaba apoyo de la CEAP. Por el contrario, buscaron presionarla demandando, en distintas reuniones, compromisos y respuestas de las autoridades y directivos de las instituciones. Realizaron protestas y desplegados, enviaron cartas a los periódicos, procuraron ir a los programas populares de radio y televisión, formaron comisiones de visita y acompañamiento de las obras; en fin, recorrieron todas las formas de lucha propias del movimiento social.

Los representantes de la comunidad también usaron el GEL para denunciar la "represión policial discriminatoria y exagerada".${ }^{\ddagger}$ El argumento presentado ante las autoridades para comprometerlas con su lucha era: "Para qué luchar por la salud de los niños, si hay la posibilidad de que la policía los mate cuando sean adolescentes." De igual forma canalizaron demandas por medio del GEL, para conseguir otros servicios públicos que inciden sobre la salud, tales como luz,, transporte, basura\& ${ }^{\&}$ teléfonos públicos.

Otra actividad desarrollada fue la de promover la organización de la comunidad. Para eso, llegaron a editar un pequeño periódico que circulaba por los centros de salud y escuelas de la región. Promovían también los Encuentros de Salud; en uno de ellos, llegaron a instalar 25 puestos, y cada uno ofrecía información sobre diferentes problemas de la comunidad-dengue, SIDA, enfermedades de transmisión sexual, la reforma sanitaria y otros-, o bien, funcionaba como tribuna

\footnotetext{
* Reunión del día 19 de septiembre de 1986.

‡ Reunión del día 20 de junio de 1987.

§ Reunión del día 13 de agosto de 1986.

\& Reunión del día 13 de septiembre de 1986.
} 
libre. Los puestos fueron organizados por la propia comunidad o por los representantes de los servicios. También promovían actividades de recreación, con teatros, bailes y música.

Para esas actividades y demandas fue importante la presencia de personas que actuaban como catalizadores de la confianza y la esperanza de la comunidad, y que colaboraban en la creación de las condiciones necesarias para la existencia del GEL. El papel de los llamados "agentes externos"15 consistía en participar como elementos que, junto con la población, debían articular y establecer lazos entre la carencia vivida y la acción propiamente dicha. Sólo así era posible explicar la transición del reconocimiento de una situación de carencia a la formulación de demandas.

La presencia de agentes externos no disminuyó la importancia de esa instancia como un espacio colectivo de construcción de prácticas ciudadanas; por el contrario, gracias a ese proceso de interacción con otros actores sociales y con el propio Estado -como poder público-, a partir de una relación ambigua y compleja, se produjeron las condiciones para la transformación de las partes presentes y de la propia relación entre Estado y sociedad.

\section{Discusión}

Mediante esta investigación fue posible recuperar la historia social y cotidiana, así como el conjunto de las demandas y necesidades en salud, de los habitantes de la periferia de una de las mayores concentraciones urbanas de Brasil. A su vez, el análisis permitió comprender el papel diferenciado que jugaron los diversos actores sociales presentes en la comunidad investigada, así como las propuestas, los consensos y conflictos en torno a la participación de la comunidad en las políticas de salud y, principalmente, las dificultades y contradicciones presentes durante la descentralización de los servicios de salud.

La investigación también capturó la dinámica del juego entre la movilización y la respuesta de los servicios de salud al hacer resaltar, por un lado, los límites y potencialidades de las propuestas del gobierno en relación con la incorporación de los grupos sociales como interlocutores y actores propositivos en la definición de demandas, y, por el otro, los marcos de la propia institucionalización de la participación popular en contextos caracterizados por la exclusión social y política.

Dicha exclusión propició prácticas clientelares y manipuladoras que condujeron a una situación de desconfianza por parte de la población respecto a los políticos y a todo lo que venía del Estado; sin embar- go, la población aceptaba la presencia y la colaboración de la Iglesia y de los profesionales de la salud de las dos instituciones universitarias que actuaban en esta localidad.

En ese sentido, la investigación permitió observar que la población, mediante los GT creados y con ayuda de los investigadores de las dos instituciones de enseñanza presentes, fue capaz de identificar algunos de los principales problemas de salud del área y proponer soluciones. A su vez, el estudio hizo posible establecer los límites y potencialidades de las propuestas gubernamentales, así como los alcances de las formas comunitarias de organización cuando actúan como interlocutores propositivos en la definición de demandas sentidas y vividas.

Esta investigación también posibilitó situar un encuentro conflictivo entre dos lógicas distintas: la de los formuladores de políticas de salud y la de la población. En ese sentido, profundizó en el conocimiento del contradictorio camino que toman los proyectos elaborados sin la participación de los actores objeto de la política y sin considerar lo que significa su aplicación cotidiana.

Para el desarrollo del GEL fueron importantes las movilizaciones constantes y el diálogo permanente con el poder público en torno de la demanda de derechos. Sin embargo, la propia percepción de la población referente a las posibilidades que el GEL tenía de influir en la práctica de los servicios de salud fue decretando lentamente su propia muerte, tema ampliamente discutido en diversas reuniones. En ellas, el tema de la representatividad retornó, al igual que el de la autonomía y el del clientelismo que establecían los partidos y otras organizaciones con el GEL.

El GEL de la AP.3.1, como un canal de participación creado por el poder público para facilitar la aplicación de una política específica, se fue transformando paulatinamente a lo largo de su existencia, de tal forma que llegó a tener características que se distanciaban de las atribuidas inicialmente. Tal fenómeno fue posible gracias a la capacidad de los grupos ahí presentes de definir, mediante la interacción con otros actores y con el poder público, un perfil de carencia y de servicios, así como una pauta de acción. Su límite fue dado por su fuerte naturaleza demandante, condición inevitable frente a una población sin las míminas condiciones de existencia. Logró expandirse mediante las conquistas inmediatas, y en la medida en que éstas fueron escaseando, el grupo fue también perdiendo su contorno.

La fragilidad del GEL radicó en su dificultad para institucionalizarse; se trató de una vulnerabilidad instrumentada por la propia comunidad. El GEL se con- 
virtió en un espacio de demanda y de lucha frente a las carencias sentidas y vividas. En vez de constituirse en una instancia de participación de la población en las decisiones y el control de los servicios, como proponían algunas organizaciones de izquierda, el GEL se transformó en un pequeño espacio político donde los habitantes pobres de la periferia pudieron, en un corto periodo, conseguir visibilidad por sí mismos.

En cierto sentido, el GEL, como instancia de participación, presentó evidencias empíricas que ponen en duda algunos de los planteamientos teóricos sobre la sociedad civil, en particular aquel que concibe a esta última como el semillero de experiencias ciudadanas e innovadoras capaces de fomentar una cultura cívica en oposición al elevado poder discrecional del Estado. ${ }^{16,17}$ Esta visión ha sustentado propuestas de participación social en salud, que han tenido el propósito de promover instituciones cívicas de base comunitaria, capaces de generar comportamientos caracterizados por una mayor iniciativa y, por lo tanto, por una menor dependencia hacia el Estado. Estas experiencias -muchas de ellas sustentadas en la autoayuda o en la búsqueda de la autosuficiencia económica-, al actuar de espaldas al Estado, lo desconocen como interlocutor legítimo en la distribución igualitaria de los bienes en sociedades marcadas por la diversidad, al igual que como actor del proceso político. Por lo tanto, se apegan a la idea de la libertad de conducta de los individuos, incluyendo sus responsabilidades, intereses previamente definidos y roles. Más que de transformar estructuras sociales, se trata de cambiar comportamientos individuales sin considerar las múltiples relaciones que se entrecruzan en las experiencias de participación popular.

Es innegable que los procesos de participación social deben buscar una autonomía en relación con el Estado, así como evitar la manipulación y el verticalismo en las relaciones. Sin embargo, no todas las experiencias incubadas en la sociedad brasileña fueron pensadas con el propósito de incentivar comportamientos de civilidad; inclusive, algunas creadas con este propósito, involucran agentes sociales que se oponen a la participación de los de "abajo" en los procesos de decisión.

El fenómeno de la participación es hoy una realidad en Brasil. No obstante, algunas organizaciones ciudadanas reproducen el padrón elitista de la sociedad brasileña, y otras se alienan de la esfera pública. La promoción de la ciudadanía es un proceso racional que se apoya en proyectos orientadores de la acción colectiva, en contextos determinados y con vistas a la construcción de relaciones sociales que tornen viables sus proyectos.
Este trabajo se situó entre aquellos que ven en la participación social un mecanismo que favorece positivamente el manejo de la salud como un bien público; ello, sin duda, requiere de ciudadanos capacitados para defender sus derechos, como condición para procesar conflictos y cambiar las situaciones de carácter desigual favorecidas por el mercado. Esto explica la insistencia de algunos autores -misma que se comparte en este trabajo- de buscar establecer una relación positiva entre participación social y poder, de tal manera que se constituya un espacio que movilice saberes y prácticas, detrás de los cuales puedan actuar agentes sociales no necesariamente unitarios y homogéneos. La propuesta es considerar estas instancias como novedosas cuando, por un lado, buscan incorporar, en las estructuras de poder del sector salud, a los que están aislados y, por otro, cuando hacen innovaciones en las formas de poder que existían antes. Estos análisis ponen énfasis en las acciones e interacciones concretas de los actores, así como en la relación cotidiana de la población con los servicios, en su percepción de las necesidades en salud y en la producción de sus demandas. ${ }^{18}$

El presente estudio coincide con los de aquellos autores que matizan los alcances de esas experiencias y resaltan la importancia de investigaciones rigurosas que las evalúen, -de tal forma que los distintos actores sociales aprendan con ellas-, que generen instrumentos orientadores de la acción de sus promotores y que produzcan, al mismo tiempo, un conocimiento de la realidad social. ${ }^{19}$ No se puede disminuir la importancia que tiene, para la eficacia de las experiencias de participación, el propio proceso que éstas engendran. Deslindarlos pasó a ser importante tanto para sus protagonistas como para los investigadores, quienes así pueden dar una dirección específica a sus reflexiones y evitar que éstas se transformen en un simple diálogo académico entre pares. ${ }^{20}$

Antonio Ugalde ${ }^{21}$ ha llamado la atención acerca del uso de la participación social en salud como un medio y no como un fin en sí mismo en muchas de las experiencias de América Latina. Para él, en este tipo de procesos sociales, o se busca cambiar en la población hábitos identificados como nocivos para su salud, o se utiliza a los pobres como apoyo en las funciones administrativas de los centros de salud, o bien, se les utiliza para poner en práctica determinados programas. Asimismo, cuando el Estado está interesado en controlar a las comunidades, la participación social conlleva el propósito de integrarlas en un proyecto que nulifica sus identidades en nombre de lo nacional. En este marco, los usos y costumbres son tomados en consideración y reconocidos como tales sólo en la me- 
dida en que sean funcionales para los objetivos de las autoridades gubernamentales.

Así, el reto para los investigadores y los promotores de estas experiencias es contribuir a la construcción de identidades ciudadanas integradas activamente en la práctica social, lo que significa, como sugiere Mouffe, constituirse en algo más que un "recipiente pasivo de derechos específicos que goza de la protección de la ley". ${ }^{22}$ La receta para eso todavía no está lista; de ahí la importancia de continuar reflexionando en torno de estas prácticas participativas y de sus relaciones con la promoción de la equidad de los servicios de salud.

\section{Agradecimientos}

Agradezco al doctor Luis Durán Arenas su estímulo y apoyo; a mis colegas, la doctora Sofía Arjonilla Alday, la maestra Irene Parada del Toro y la maestra Isabel Hernández Tezoquipa, sus lecturas, y a los editores y revisores, sus comentarios acertados. Todos permitieron mejorar este artículo que, sin embargo, es de absoluta responsabilidad de la autora.

\section{Referencias}

I. Medici A M. A dinâmica do setor saúde no Brasil -Transformações e tendencias nas décadas de 80 e 90. Santiago de Chile: Cepal, 1997.

2. Gerschman S. A democracia inconclusa -Um estudo da reforma sanitária brasileira. Río de Janeiro: Editora Fiocruz, 1995.

3. Cardoso R, comp. A aventura antropológica. Teoría e pesquisa. 2a. edición. Brasil: Paz e Terra, 1989.

4. Muro G, Canto-Cãc M, eds. El estudio de los moviemientos sociales. Teoría y Método. México, D.F.: El Colegio de Michoacán-UAM, |99|.

5. Machado da Silva L. Para pensar o paradigma dos movimientos sociais urbanos. X Encontro da Associaçao Nacional de Pós-Graduação e Pesquisa em Ciênciás Sociais. Sao Paulo: Campos do Jordão, 1986.
6. Cohen J, Touraine A, Melucci A, Jenkins JC. Teoría de los movimientos sociales. San José de Costa Rica: Flacso (Cuadernos de Ciencias Sociales), 1988.

7. Munck G. Estratégia Política: problemas conceítuais do estudo dos movimientos sociales. Dados 1997;40(7):105-126.

8. Guba E, Lincoln Y. Competing paradigms in qualitative research. Handbook of qualitative research. Nueva York: Sage Publications, 1994.

9. O'Donnell G, Schmitter P. Transição do regime autoritário. Primeiras conclusões. Sao Paulo: Editora Vértice, 1988.

10. Sallum Jr. B. Labirintos. Dos generais à Nova República. Sao Paulo: Hucitec, 1996.

II. Cordeiro H. Sistema Único de Saúde. Río de Janeiro: Ayuri Editorial, 1991.

12. Costa NR, Minayo C, Ramos C, Stotz E, eds. Demandas populares, políticas públicas e saúde. Petrópolis: Vozes, 1989.

13. Abrantes R. Movimentos sociais na saúde e identidades colectivas: Um estudo de caso. En: Bodstein R. Serviços locais de saúde: construção de atores e políticas. Rio de Janeiro: Relume Dumará, 1993.

14. Stotz E. Luta pela saúde ambiental: a AMAP contra Curtume Carioca S.A. Uma experiência vitoriosa. En: Valla V, Stotz E. Participação popular, educação e saúde: teoria e prática. Río de Janeiro: Relume Dumará, 1993: 133-160.

15. Jacobi P. Movimentos sociais e políticas públicas: demandas por saneamento básico e saúde. São Paulo, 1974-84. São Paulo: Cortez, 1989. 16. Kymlicka W, Norman W. El retorno del ciudadano. Una revisión de la producción reciente en teoría de la ciudadanía. La política 1997;(3):5-40. 17. Hommes R. Conflicts and dilemmas of decentralization. En: Annual Bank Conference on Development Economics; 1995 mayo; Washington D.C., EUA.

18. Cohn A. Saúde e ciudadania: análise de uma experiencia de gestão local. En: Eibenschutz. Política de saúde: o público e o privado. Río de Janeiro: Editora Fiocruz, 1995.

19. Haro y Keijzer A, comp. Participación comunitaria en salud: evaluación de experiencias y tareas para el futuro. México: El Colegio de Sonora, 1998.

20. Schteingart M. La investigación-acción y las relaciones entre organizaciones no gubernamentales y sector académico. En: Méndez JL. Organizaciones civiles y políticas públicas en México y Centroamérica. México, D.F.: Porrúa, 1998:63-75.

21. Ugalde A. Las dimensiones ideológicas de la participación comunitaria en los programas de salud en Latinoamérica. Cuadernos Médicos Sociales (Buenos Aires) 1987; (4I):27-43.

22. Mouffe C. Ciudadanía democrática y comunidad política. En: Buenfil Debates políticos contemporáneos -En los márgenes de la modernidad. México, D.F.:PyV editores: 138. 